\title{
Random-matrix perspective on many-body entanglement with a finite localization length
}

\author{
Marcin Szyniszewski $\oplus^{1,2}$ and Henning Schomerus $\oplus^{1}$ \\ ${ }^{1}$ Department of Physics, Lancaster University, Lancaster LA1 4YB, United Kingdom \\ ${ }^{2}$ Department of Physics and Astronomy, University College London, London WC1E 6BT, United Kingdom
}

(Received 26 October 2019; accepted 25 June 2020; published 8 July 2020)

\begin{abstract}
We provide a simple and predictive random-matrix framework that naturally generalizes Page's law for ergodic many-body systems by incorporating a finite entanglement localization length. By comparing a highly structured one-dimensional model to a completely unstructured model and a physical system, we uncover a remarkable degree of universality, suggesting that the effective localization length is a universal combination of model parameters up until it drops down to the microscopic scale.
\end{abstract}

DOI: 10.1103/PhysRevResearch.2.032010

Introduction. In this Rapid Communication we present a generalization of Page's law [1] — central to the statistical description of entanglement in completely ergodic many-body systems - so that it incorporates a finite entanglement length scale, designed to represent an effective localization length in a many-body localized system [2-6]. Page's law is based on the simple assumption that a typical ergodic many-body eigenstate $|\psi\rangle$ constitutes a random Fock-space vector with independent identically distributed Gaussian entries $\psi_{m}=$ $\langle m \mid \psi\rangle$. Bipartitioning the system as a tensor product $|m\rangle=$ $|a b\rangle$, with indices $a=1,2,3, \ldots, M_{A}$ for a subsystem $A$ and $b=1,2,3, \ldots, M_{B}$ for its complement $B$, the reduced density matrix $\rho_{a a^{\prime}}^{A \mid B}$ of a subsystem $A$ can be reinterpreted as a matrix product,

$$
\rho^{A \mid B}=\frac{V V^{\dagger}}{\operatorname{tr} V V^{\dagger}},
$$

where $V_{a b}=\langle a b \mid \psi\rangle$ is a random $M_{A} \times M_{B}$ Gaussian matrix. This ties the description to the celebrated Wishart ensemble of random matrix theory - the inaugural ensemble of random matrix theory in the history of science [7], which is based on completely positive Hermitian matrices of the form $V V^{\dagger}$. Applying these arguments, Page then arrived at the prediction

$$
S(A \mid B)=-\operatorname{tr}\left(\rho^{A \mid B} \ln \rho^{A \mid B}\right)=\ln M_{A}-\frac{M_{A}}{2 M_{B}}
$$

for the ensemble-averaged bipartite von Neumann entanglement entropy, assuming $1 \ll M_{A} \leqslant M_{B}$ [8]. This prediction serves as an important benchmark to detect deviations from ergodic many-body behavior, including signatures of manybody localization and topological states [9-13].

Here, we present a simple and predictive statistical framework that accurately captures these deviations, and covers the

Published by the American Physical Society under the terms of the Creative Commons Attribution 4.0 International license. Further distribution of this work must maintain attribution to the author(s) and the published article's title, journal citation, and DOI. distance from entirely ergodic behavior to the strongly manybody localized regime. This gives very direct and specific insights into a transition that so far has been addressed mainly through insightful perturbative strong-disorder renormalization schemes [14-19]. We first give a simple motivation and description of the framework and analyze its main features, amongst which is a surprising degree of universality with regard to both the microscopic parameters as well as the local structure of the random-matrix model. We then demonstrate its predictive power in comparison with a paradigmatic spinchain model. Finally, we discuss the framework from the general perspective of matrix-product states.

Premise and background. To motivate our approach it is suggestive to declare Page's statistical assumptions as natural in the following sense: The matrix $V$ can be interpreted to capture the correlation amplitudes between the adjacent parts $A$ and $B$, in a statistical invariant way where, for instance, any independent superposition $V=\sum_{\alpha=1}^{N_{\alpha}} V^{\alpha}$ of matrices from the same Gaussian ensemble delivers the same statistics [20]. In our generalization, the system is partitioned into a larger number of small ergodic patches $P_{1}, P_{2}, P_{3}, \ldots$, which we take of identical dimensionality $M_{0}$, and the wave function takes the simple form

$$
\psi_{a b c d \ldots}=\sum_{\alpha=1}^{N_{\alpha}} V_{a b}^{1 \mid 2, \alpha} V_{b c}^{2 \mid 3, \alpha} V_{c d}^{3 \mid 4, \alpha} \ldots
$$

The random Gaussian matrices $V^{k \mid k+1, \alpha}$ again describe the correlations between neighboring ergodic patches, only that there are now many of these. This defines the highly structured variant of our model. Taking, in contrast, the matrices $V$ as separable, we arrive at a completely unstructured model equivalent to a superposition of completely separable states

$$
\psi_{a b c d \ldots}=\sum_{\alpha=1}^{N_{\alpha}} \chi_{a}^{1, \alpha} \chi_{b}^{2, \alpha} \chi_{c}^{3, \alpha} \chi_{d}^{4, \alpha} \ldots
$$

with random amplitudes $\chi$, which is agnostic about the ordering of the patches and hence does not contain any information about geometric features, such as dimensionality and bound- 

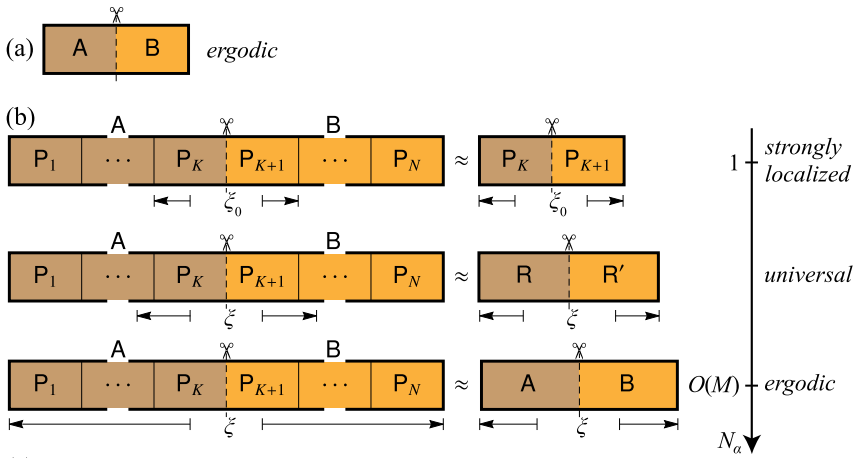

(c)

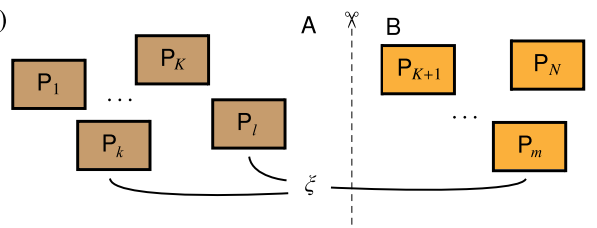

FIG. 1. (a) Page's law (2) describes the entanglement in an ergodic system partitioned into parts $A$ and $B$. (b) The highly structured model (3) describes the transition from a strongly localized regime, where the bipartitioned system can be effectively reduced to two small ergodic patches next to the partition point, over a universal regime with a finite localization length (5), to the ergodic case where Page's law is recovered. (c) In the completely unstructured variant (4), a similar transition occurs but the ergodic regions may be interpreted as noncontiguous.

ary conditions. The interplay of models (3) and (4) defines our random-matrix framework. In both cases, the reduced density matrix for a bipartition $A\left|B=P_{1} \ldots P_{K}\right| P_{K+1} P_{K+2} \ldots$ is obtained by tracing out the sequence of patches $P_{K+1} P_{K+2} \ldots$.

We will argue, and verify numerically, that this framework identifies key entanglement characteristics of systems with a finite range of the entanglement, subsumed into a universal effective localization length $\xi$ that combines the microscopic model parameters $M_{0}$ and $N_{\alpha}$ into one. The universality is fully established in the mesoscopic regime, where the parts are all small compared to the size of the bipartioned subsystems, and $N_{\alpha}$ is moderately large, but in practice already holds well for $N_{\alpha}=O(1)$. In particular, in comparison to physical models the framework turns out to be remarkably predictive for the bipartite entanglement entropy at different system sizes and choice of bipartition [11,12,21,22]. As this universality is observed also between the two variants of the model, we conjecture that it also extends to interpolating scenarios, including multifractal cases [23].

The key idea of the model, namely, partitioning the system into small patches $P_{k}$ of size below the universal localization length scale and then considering superpositions to arrive at a universal mesoscopic regime, is shown pictorially in Fig. 1. A complementary approach has been taken before by several groups [14-19], who set up insightful perturbative strongdisorder renormalization schemes for many-body localized systems based on coupling strengths and thermalization rates between coupled blocks. In contrast, our statistical approach directly stipulates the wave functions of the composed system. Conceptually, this wave-function centered construction starting from the ergodic limit has its precedent in powerful approaches to single-particle Anderson localization. In in- fluential papers by Dorokhov, Mello, Pererya, and Kumar (DMPK) $[24,25]$ it has been shown that Anderson localization naturally arises from the multiple scattering in a chain of individual weakly scattering components. Analogously, Iida, Weidenmüller, and Zuk (IWZ) [26] showed that the same universal behavior emerges from the multiple scattering in a chain of individually strongly scattering components. The DMPK model and the IWZ model both attain the same universal thick-wire fixed point as the supersymmetric $\sigma$ model [27,28], which is governed by a single length scale, the single-particle localization length, in accordance to the oneparameter scaling hypothesis [29]. The model and predictions presented in our work can serve as a benchmark to establish to which extent an analogous form of one-parameter scaling applies to many-body localization. For establishing a gradually more ergodic behavior of wave functions by superpositions, a useful reference point is Berry's random wave model for quantum-chaotic systems [30], which we here effectively carry over to Fock space based on wave functions equipped with a suitable local structure. With this wave-functioncentered approach, the resulting framework complements the application of random-matrix theory as a benchmark for energy-level statistics $[3,4,31,32]$. Other phenomenological approaches recently gained substantial interest, e.g., in systems with imposed entanglement restrictions [33], as they provide simple yet predictive pictures of phenomena that are otherwise difficult to describe microscopically.

Key features. To identify the key features of the highly structured model (3) and the completely unstructured model (4), Fig. 2 shows the bipartite entanglement entropy obtained in systems of different length and patch size. For definiteness, we phrase the length scales in the language of systems with $N$ spins, broken down into small patches of length $N_{0}$ (thus $\left.M_{0}=2^{N_{0}}\right)$.

In the left panels, we vary the partition point while keeping $N_{\alpha}$ fixed. For $N_{\alpha}=O(1)$, the entropy is small and independent of the partition point, corresponding to a highly localized system. For increasing $N_{\alpha}$, the entropy rises, and finally attains the ergodic result (2) for the complete system, which now depends on the partition point. Remarkably, the curves of both models match up closely in the crossover.

We will reveal below by statistical arguments that this amounts to a universal behavior governed by a single parameter in each model, an effective localization length

$$
\xi \sim \xi_{0}+2 \log _{2} N_{\alpha} .
$$

In particular, for $N_{\alpha}=1$ the entropy in the structured model closely conforms to the ergodic result (2) for a reduced system with only two patches adjacent to the partition point, hence effective localization length $\xi=\xi_{0}=2 N_{0}$. In the unstructured model, the entropy vanishes in this limit, so that $\xi_{0}=$ 0 . Increasing $N_{\alpha}$ then amounts to gradually increasing the effective range of ergodic behavior, with a universal scaling of the effective localization length. This universal scaling is verified in the right panels, where we keep the partition point fixed. We see that the universal scaling is attained quickly for moderately large patch and system sizes.

Application to physical models. Below, we will give a detailed statistical justification of this universal behavior. First, we describe how it conforms and applies to concrete physical 

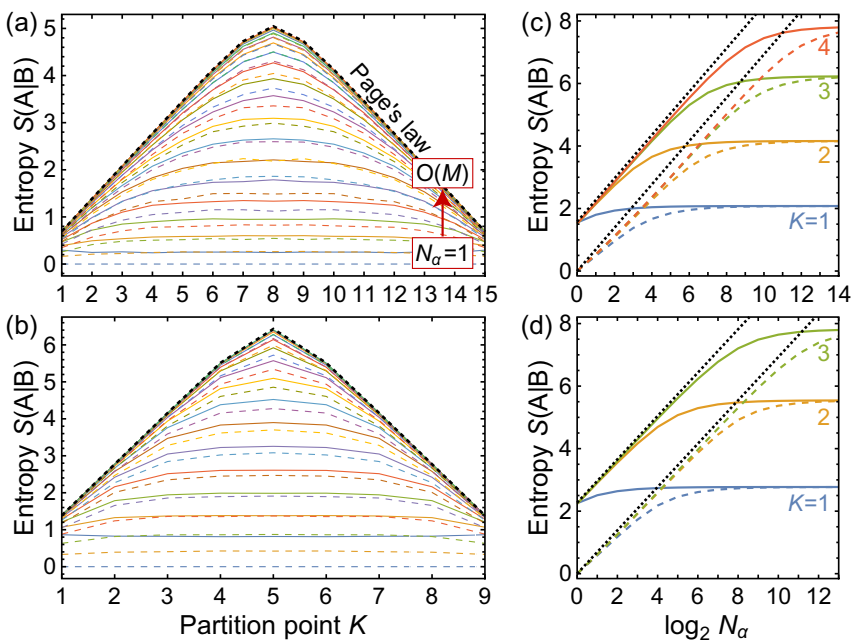

FIG. 2. Bipartite entanglement entropy $S(A \mid B)$ as predicted by the structured model (3) (solid curves) and the unstructured model (4) (dashed curves), for different partitions $A\left|B=P_{1} \ldots P_{K}\right| P_{K+1} \ldots$, where each patch has $2^{N_{0}}$ internal states. In (a) the system is made of 16 patches of size $N_{0}=1$, in (b) of 10 patches of size $N_{0}=2$, in (c) of 8 patches of size $N_{0}=3$, and in (d) of 6 patches of size $N_{0}=4$. Panels (a) and (b) highlight the dependence with the partition point for different numbers of superimposed states $N_{\alpha}=2^{m}, m=$ $0,1,2 \ldots$, where the results for large $m$ approach the ergodic result from Page's law (2) (thick dotted curve). Panels (c) and (d) highlight the dependence on $N_{\alpha}$, where the sloped dotted lines correspond to an ergodic system truncated to the effective localization length (5); for large $N_{\alpha}$ the curves level off at Page's law. We find excellent agreement with the predicted universal behavior, which sets in quickly for increasing patch and system size.

systems. This is illustrated in Fig. 3, where we provide a comparison to results for a spin chain with Hamiltonian

$$
\hat{H}=\sum_{n} \mathbf{h}_{n} \cdot \boldsymbol{\sigma}_{n}-\sum_{n} \boldsymbol{\sigma}_{n} \cdot \boldsymbol{\sigma}_{n+1},
$$

where $\sigma_{n}$ is a vector of Pauli matrices on the $n$th site, and $\mathbf{h}_{n}=$ $\left(h_{n}^{x}, h_{n}^{y}, h_{n}^{z}\right)$ describes a random field with coefficients drawn independently from a uniform distribution over $[-W, W]$.

The results in the figure are averaged over 1000 realizations of the disorder. They are compared to the random-matrix models (3) and (4) with patch size $N_{0}=1$ (corresponding to individual spins, hence allowing us to reach small localization lengths) and selected values of $N_{\alpha}$, also averaged over 1000 realizations. The values of $N_{\alpha}$ are chosen by matching the random-matrix models to the spin-chain result at the equal bipartition (center of the curve), and kept fixed for the other partition points. In panel (a) we only take states in the middle of the spectrum (central $10 \%$ of states in each realization) and vary the disorder strength, while in panels (b) we fix the disorder strength and vary the energy range (separating the states in each realization by energy into ten groups, each containing $10 \%$ of the states). In all cases, the entropy varies consistently with choice of the partition point, disorder strength, and energy range, and is in excellent agreement with the random-matrix models (3) and (4). As illustrated in panels (c) and (d), this allows one to determine the effective localization length, Eq. (5), from the data of the physical
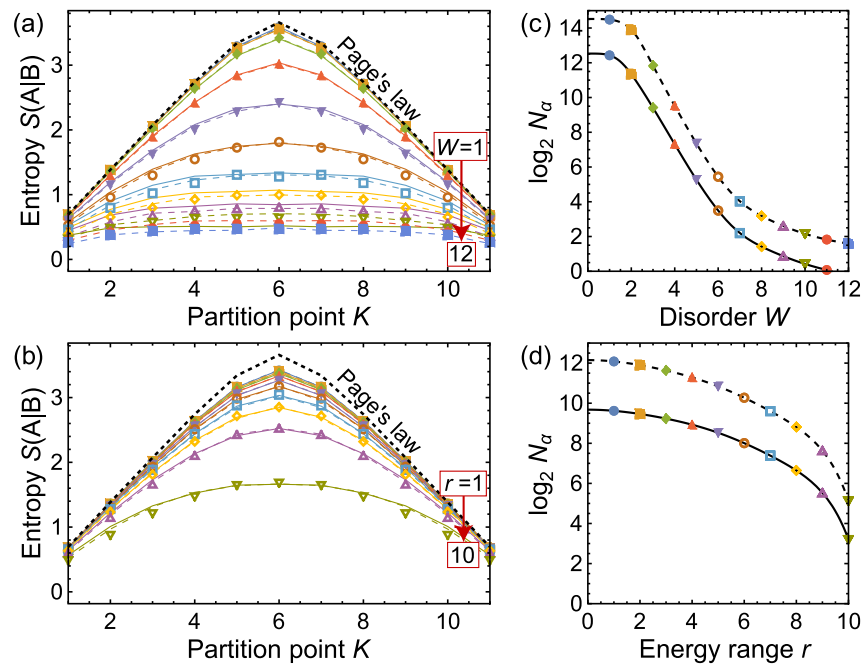

FIG. 3. (a),(b) The markers show the bipartite entanglement entropy in spin-chain (6) with $N=12$ sites as a function of the partition point $K$, for (a) different strengths of disorder $W=1,2, \ldots, 12$ obtained from the $10 \%$ of states closest to the band center, or (b) at fixed disorder strength $W=3$ with the states separated by energy into ten groups ranging from the band center (where the entropy is large, range $r=1$ ) to the band edge (where it is small, $r=10$ ). The thick dashed curve indicates Page's law (2) for the ergodic limit, while the thin solid and dashed curves show the corresponding predictions from the random-matrix models (3) and (4) in analogy to Fig. 2, with patch size $N_{0}=1$. In (c) and (d), the corresponding values of $\log _{2}\left(N_{\alpha}\right)$ are plotted for the same system size as a function of disorder strength or energy range, which delivers the effective localization length (5).

model, which is one of the key merits of our approach. This universal value is independent of the partition. The sudden drop of the effective localization length occurs near $W \approx 5$, which agrees well with the transition point to the many-body localized behavior in the literature [34,35].

Statistical justification of universality. The observed universal behavior in the random-matrix models (3) and (4) follows directly from the statistical properties of the framework. These are subsumed into two key features: (i) the self-averaging property

$$
\sum_{b} V_{a b}^{k \mid k+1}\left(V_{a^{\prime} b}^{k \mid k+1}\right)^{*} \rightarrow \delta_{a a^{\prime}},
$$

quickly valid from a moderate number of terms in the sum, and (ii) the fact that wave functions drawn from each model constitute a statistically complete basis,

$$
\overline{|\psi\rangle\langle\psi|}=\mathbb{1} .
$$

In particular, in the structured model (3), we can use the self-averaging property to show that for $N_{\alpha}=1$ the entropy of a partition $A\left|B=P_{1} \ldots P_{K}\right| P_{K+1} P_{K+2} \ldots$ reduces to that of the patches adjacent to the partitioning point,

$$
S(A \mid B) \approx S\left(P_{K} \mid P_{K+1}\right),
$$


which in turn is given by Page's result (2) for the reduced system. To see this, let us write the wave function (3) for $N_{\alpha}=1$ as

$$
\psi_{\mathbf{a} a_{K} b_{K+1} \mathbf{b}}=\psi_{\mathbf{a}, a_{K}}^{(A)} V_{a_{K} b_{K+1}} \psi_{b_{K+1}, \mathbf{b}}^{(B)}
$$

where $a_{K}$ and $b_{K+1}$ are the indices in the patches next to the partition point, with Gaussian correlation amplitudes $V \equiv$ $V^{K \mid K+1}$, while a and $\mathbf{b}$ subsume all the other indices (we also drop the index $\alpha$ ). The structure implies that the reduced density matrices $\rho^{A \mid B}$ have the same rank as the truncated density matrix $\rho^{P_{K} \mid P_{K+1}} \propto V V^{\dagger}$ : In the space of indices $\mathbf{a}$, any state orthogonal to the span of $\psi_{\mathbf{a}, a_{K}}^{(A)}$ corresponds to a vanishing eigenvalue. Furthermore, to a very good approximation both matrices share the same entanglement spectrum: The self-averaging property (7) implies $\sum_{\mathbf{a}} \psi_{\mathbf{a}, a_{K}}^{(A)} \psi_{\mathbf{a}, a_{K}^{\prime}}^{*(A)} \rightarrow$ $\delta_{a_{K} a_{K}^{\prime}}$. Hence, the finite eigenvalues of $\rho^{A \mid B}$ are recovered with high accuracy from approximate eigenvectors $\varphi_{\mathbf{a} a_{K}}=$ $\psi_{\mathbf{a}, a_{K}}^{(A)} \varphi_{a_{K}}$ where $\boldsymbol{\varphi}$ is an eigenvector of $\rho^{P_{K} \mid P_{K+1}}$. Thereby, the entropy is given by Page's result for the reduced system of only two adjacent patches, as stipulated in Eq. (9).

For a finite number $N_{\alpha}$ of states participating in Eq. (3), a similar self-averaging argument applies to show that

$$
S\left(P_{1} \ldots P_{K} \mid P_{K+1} P_{K+2} \ldots\right) \approx S\left(R \mid R^{\prime}\right),
$$

with effective ergodic patches $R, R^{\prime}$ of increased size $N_{0}+$ $\log _{2} N_{\alpha}$, hence a reduced system of overall size as given by Eq. (5). Here we use that the collection of states $\psi_{\mathbf{a}, a_{K}}^{(A) \alpha}$ with reinstated label $\alpha$ remains statistically orthogonal to each other as long as $N_{\alpha}$ does not grow too large. Thereupon, $\rho^{A \mid B}$ shares the entanglement spectrum of a direct sum of matrices $N_{\alpha}^{-1} \bigoplus_{\alpha=1}^{N_{\alpha}} V^{\alpha} V^{\alpha \dagger}$. Accounting also for the indicated overall normalization, the entropy then increases by $\log _{2} N_{\alpha}$, resulting in Eq. (11).

Establishing the ergodic behavior for large $N_{\alpha}$ is equally straightforward. Even if matrices $V^{\alpha}$ were drawn from a highly structured distribution, the Wishart ensemble underlying Page's result is recovered from $V=\sum_{\alpha} V^{\alpha}$ for large $N_{\alpha}$ as long as the matrices form a complete basis in a statistical sense. This applies, in particular, also to independently drawn states of the form (3), whose span covers the whole space according to their ensemble average (8). Adding a large number $N_{\alpha}=O(M)=O\left(2^{N}\right)$ of these states therefore recovers the ergodic case. This expectation is again compatible with the logarithmic growth of the effective localization length (5) stipulated above.

For the unstructured model, the same arguments can be adapted in a simplified form. For $N_{\alpha}=1$, the entropy vanishes, corresponding to $\xi_{0}=0$. For moderate values of $N_{\alpha}$, we observe the same statistical direct sum as above, giving rise to the logarithmic scaling, until one reaches the ergodic limit where we can again utilize that states drawn from Eq. (4) form a statistically complete basis.

Relation to matrix-product states. To further illuminate our framework we connect it to the general framework of matrix-product states. This framework provides a universal representation of many-body states, and arises mathematically from successive applications of singular-value decompositions [36], which are also at the heart of the analysis of the Wishart ensemble [37,38]. Starting from Eq. (3), we can make this connection explicit by using a singular-value decomposition of the correlation matrices,

$$
V_{a b}^{k \mid k+1, \alpha}=\sum_{l} u_{a l}^{k, \alpha} \lambda_{l}^{k \mid k+1, \alpha} v_{l b}^{k+1, \alpha},
$$

with unitary matrices $u^{K, \alpha}$ and $v^{K, \alpha}$ and diagonal matrices $\lambda$ of Schmidt coefficients (encoding the entanglement spectrum of the reduced system with patches $P_{K}$ and $\left.P_{K+1}\right)$. Rearranging terms and reinterpreting indices, this directly delivers the representation

$$
\psi_{a b c d \ldots}=\operatorname{tr}\left(\Gamma^{1, a} \Lambda^{1 \mid 2} \Gamma^{2, b} \Lambda^{2 \mid 3} \ldots\right),
$$

with block matrices $\Gamma_{l m}^{k, a}=\bigoplus_{\alpha} v_{l a}^{k, \alpha} u_{a m}^{k, \alpha}$ and $\Lambda^{k \mid k+1}=$ $\bigoplus_{\alpha} \lambda^{k \mid k+1, \alpha}$. Equation (13) is analogous to Vidal's representation of matrix-product states [39], which naturally incorporates entanglement characteristics in the diagonal matrices $\Lambda$, but with two notable practical differences. (i) In Vidal's representation, the matrices $\Lambda^{k \mid k+1}$ contain the exact entanglement spectrum of the bipartition $A \mid B$ of the complete system, while here they contain the approximate entanglement spectra of the neighboring patches. However, both spectra accurately agree according to our derivation of Eq. (9). In spirit, this conforms to the conventional reduction of matrix-product states by truncation of the entanglement spectrum, hence the rank of matrices $\Lambda$ [40]. (ii) Canonically, Vidal's representation is designed to describe a single state, while the block structure above implies this representation being carried out for each individual state in the sum over $\alpha$. Thereby, we here also encounter a truncation of the matrices $\Gamma$, which are of $\operatorname{rank} N_{\alpha}$.

In principle, our framework can therefore also be formulated in the language of matrix-product states. Arguably, however, the two modifications outlined above cannot be easily anticipated without the guidance of the physically transparent form (3) of the underlying wave function. As shown above, it is indeed the interplay of these two truncations which sets the universal entanglement length scale (5).

Conclusions. In summary, we proposed a random-matrix framework for many-body quantum systems that captures the effect of finitely ranged entanglement, subsumed into a universal effective entanglement localization length. The framework allows us to make predictions for the entanglement entropy for different choice of partitions, which agree well with those of physical systems with different disorder strengths and energy densities. This provides a route to extract this effective entanglement localization length from data.

Just as Page's law can be utilized as a benchmark to detect deviations from ergodic behavior, the models presented here can serve as a useful benchmark to test concrete hypotheses about disordered many-body systems. For instance, while the models do not predict an entanglement localization transition in a sufficiently disordered system, or discriminate such a transition from a crossover, they can be employed to investigate this issue based on the described extraction of the effective localization length. 
More broadly, our framework incorporates a form of oneparameter scaling, and hence also allows one to test this as a hypothesis and detect possible deviations. In particular, in the structured variant of the model the effective localization length denotes a contiguous ergodic region, while the spatial structure of the ergodic region is not prescribed in the unstructured model, emphasizing the universality of this aspect over a range of possible microscopic models. The observed universality can be conjectured to extend to interpolating scenarios, including multifractal scenarios for which no simple model exists. This connects our approach directly to a crucial question in the analysis of the many-body localization transition [14-19], which can be further pursued by considering entanglement in disjoint partitions of the system [41].

All relevant data present in this publication can be accessed at Ref. [42].

Acknowledgments. This research was funded by UK Engineering and Physical Sciences Research Council (EPSRC) via Grant No. EP/P010180/1. Computer time was provided by Lancaster University's High-End Computing facility. M.S. was also funded by the European Research Council (ERC) under the European Union's Horizon 2020 Research and Innovation programme (Grant agreement No. 853368).
[1] D. N. Page, Average Entropy of a Subsystem, Phys. Rev. Lett. 71, 1291 (1993).

[2] D. M. Basko, I. L. Aleiner, and B. L. Altshuler, Metalinsulator transition in a weakly interacting many-electron system with localized single-particle states, Ann. Phys. 321, 1126 (2006).

[3] E. Altman and R. Vosk, Universal dynamics and renormalization in many-body-localized systems, Annu. Rev. Condens. Matter Phys. 6, 383 (2015).

[4] R. Nandkishore and D. A. Huse, Many-body localization and thermalization in quantum statistical mechanics, Annu. Rev. Condens. Matter Phys. 6, 15 (2015).

[5] D. A. Abanin and Z. Papić, Recent progress in many-body localization, Ann. Phys. (Berlin) 529, 1700169 (2017).

[6] F. Alet and N. Laflorencie, Many-body localization: An introduction and selected topics, C. R. Phys. 19, 498 (2018).

[7] J. Wishart, The generalised product moment distribution in samples from a normal multivariate population, Biometrika 20A, 32 (1928).

[8] In this limit, the result is independent of the universality class of random-matrix theory.

[9] B. Bauer and C. Nayak, Area laws in a many-body localized state and its implications for topological order, J. Stat. Mech.: Theory Exp. (2013) P09005.

[10] J. A. Kjäll, J. H. Bardarson, and F. Pollmann, Many-Body Localization in a Disordered Quantum Ising Chain, Phys. Rev. Lett. 113, 107204 (2014).

[11] L. Vidmar and M. Rigol, Entanglement Entropy of Eigenstates of Quantum Chaotic Hamiltonians, Phys. Rev. Lett. 119, 220603 (2017).

[12] T. LeBlond, K. Mallayya, L. Vidmar, and M. Rigol, Entanglement and matrix elements of observables in interacting integrable systems, Phys. Rev. E 100, 062134 (2019).

[13] V. Khemani, S. P. Lim, D. N. Sheng, and D. A. Huse, Critical Properties of the Many-Body Localization Transition, Phys. Rev. X 7, 021013 (2017).

[14] A. C. Potter, R. Vasseur, and S. A. Parameswaran, Universal Properties of Many-Body Delocalization Transitions, Phys. Rev. X 5, 031033 (2015).

[15] R. Vosk, D. A. Huse, and E. Altman, Theory of the Many-Body Localization Transition in One-Dimensional Systems, Phys. Rev. X 5, 031032 (2015).
[16] L. Zhang, B. Zhao, T. Devakul, and D. A. Huse, Many-body localization phase transition: A simplified strong-randomness approximate renormalization group, Phys. Rev. B 93, 224201 (2016).

[17] P. T. Dumitrescu, R. Vasseur, and A. C. Potter, Scaling Theory of Entanglement at the Many-Body Localization Transition, Phys. Rev. Lett. 119, 110604 (2017).

[18] T. Thiery, F. Huveneers, M. Müller, and W. De Roeck, ManyBody Delocalization as a Quantum Avalanche, Phys. Rev. Lett. 121, 140601 (2018)

[19] A. Goremykina, R. Vasseur, and M. Serbyn, Analytically Solvable Renormalization Group for the Many-Body Localization Transition, Phys. Rev. Lett. 122, 040601 (2019).

[20] Under very general conditions the central-limit theorem guarantees that even if the $V^{\alpha}$,s were to be taken from a highly structured distribution (but forming a complete basis in a statistical sense), the ergodic behavior would be recovered for large $N_{\alpha}$. This feature is exploited in the ergodic limit of our model.

[21] A. M. Kaufman, M. E. Tai, A. Lukin, M. Rispoli, R. Schittko, P. M. Preiss, and M. Greiner, Quantum thermalization through entanglement in an isolated many-body system, Science 353, 794 (2016).

[22] O. Shtanko, Y. A. Kharkov, L. P. García-Pintos, and A. V. Gorshkov, Classical models of entanglement in monitored random circuits, arXiv:2004.06736.

[23] By modifying the matrices $V^{k \mid k+1, \alpha}$ gradually it is in principle possible to interpolate between models (3) and (4).

[24] O. N. Dorokhov, Transmission coefficient and the localization length of an electron in $\mathrm{N}$ bound disordered chains, JETP Lett. 36, 318 (1982).

[25] P. A. Mello, P. Pereyra, and N. Kumar, Macroscopic approach to multichannel disordered conductors, Ann. Phys. 181, 290 (1988).

[26] S. Iida, H. A. Weidenmüller, and J. A. Zuk, Statistical scattering theory, the supersymmetry method and universal conductance fluctuations, Ann. Phys. 200, 219 (1990).

[27] K. Efetov, Supersymmetry in Disorder and Chaos (Cambridge University Press, Cambridge, UK, 1996).

[28] C. W. J. Beenakker, Random-matrix theory of quantum transport, Rev. Mod. Phys. 69, 731 (1997).

[29] E. Abrahams, P. W. Anderson, D. C. Licciardello, and T. V. Ramakrishnan, Scaling Theory of Localization: Absence of 
Quantum Diffusion in Two Dimensions, Phys. Rev. Lett. 42, 673 (1979).

[30] M. V. Berry, Regular and irregular semiclassical wavefunctions, J. Phys. A: Math. Gen. 10, 2083 (1977).

[31] V. Oganesyan and D. A. Huse, Localization of interacting fermions at high temperature, Phys. Rev. B 75, 155111 (2007).

[32] J. Šuntajs, J. Bonča, T. Prosen, and L. Vidmar, Quantum chaos challenges many-body localization, arXiv:1905.06345.

[33] S. C. Morampudi, A. Chandran, and C. R. Laumann, Universal Entanglement of Typical States in Constrained Systems, Phys. Rev. Lett. 124, 050602 (2020).

[34] S. D. Geraedts, R. Nandkishore, and N. Regnault, Many-body localization and thermalization: Insights from the entanglement spectrum, Phys. Rev. B 93, 174202 (2016).

[35] Note that the model in Ref. [34] is based on spin operators $\mathbf{S}=$ $\sigma / 2$, so that the definition of the field $h$ differs from ours by a factor of 2 .

[36] U. Schollwöck, The density-matrix renormalization group in the age of matrix product states, Ann. Phys. 326, 96 (2011).
[37] K. Życzkowski and H.-J. Sommers, Induced measures in the space of mixed quantum states, J. Phys. A: Math. Gen. 34, 711 (2001).

[38] S. N. Majumdar, Extreme eigenvalues of Wishart matrices and entangled bipartite system, in The Oxford Handbook of Random Matrix Theory, edited by G. Akemann, J. Baik, and P. Di Francesco (Oxford University Press, Oxford, 2011), Chap. 37.

[39] G. Vidal, Efficient Classical Simulation of Slightly Entangled Quantum Computations, Phys. Rev. Lett. 91, 147902 (2003).

[40] In Vidal's representation the matrices $\Gamma$ obey a specific normalization condition, which in our model is observed upon self-averaging.

[41] L. Herviou, S. Bera, and J. H. Bardarson, Multiscale entanglement clusters at the many-body localization phase transition, Phys. Rev. B 99, 134205 (2019).

[42] M. Szyniszewski and H. Schomerus, Research data for "Random-matrix perspective on many-body entanglement with a finite localization length" https://dx.doi.org/10.17635/ lancaster/researchdata/374 (2020). 